\title{
Modeling FOG Drift Using Back-Propagation Neural Network Optimized by Artificial Fish Swarm Algorithm
}

\author{
Rui Song, ${ }^{1}$ Xiyuan Chen, ${ }^{1}$ Chong Shen, ${ }^{1,2}$ and Hong Zhang ${ }^{1}$ \\ ${ }^{1}$ School of Instrument Science and Engineering, Key Laboratory of Micro-Inertial Instrument and Advanced Navigation Technology, \\ Ministry of Education, Southeast University, Nanjing 210096, China \\ ${ }^{2}$ School of Instrument and Electronics, North University of China, Taiyuan 030051, China
}

Correspondence should be addressed to Xiyuan Chen; chxiyuan@seu.edu.cn

Received 8 April 2014; Revised 13 July 2014; Accepted 14 July 2014; Published 4 August 2014

Academic Editor: Xinyong Dong

Copyright (C) 2014 Rui Song et al. This is an open access article distributed under the Creative Commons Attribution License, which permits unrestricted use, distribution, and reproduction in any medium, provided the original work is properly cited.

\begin{abstract}
Based on the temperature drift characteristic of fiber optic gyroscope (FOG), a novel modeling and compensation method which integrated the artificial fish swarm algorithm (AFSA) and back-propagation (BP) neural network is proposed to improve the output accuracy of FOG and the precision of inertial navigation system. In this paper, AFSA is used to optimize the weights and threshold of BP neural network which determine precision of the model directly. In order to verify the effectiveness of the proposed algorithm, the predicted results of BP optimized by genetic algorithm (GA) and AFSA are compared and a quantitative evaluation of compensation results is analyzed by Allan variance. The comparison result illustrated the main error sources and the sinusoidal noises in the FOG output signal are reduced by about 50\%. Therefore, the proposed modeling method can be used to improve the FOG precision.
\end{abstract}

\section{Introduction}

FOG is one kind of inertial sensors, which is based on the Sagnac effect and has been widely used in inertial system and engineering application at present. The surrounding environment of FOG is often accompanied with a wide temperature range or a fast temperature change rate. Besides, the optical components of FOG are sensitive to the environment temperature change, which will bring errors to the output of FOG [1]. Considering the complexity and difficulty of FOG's internal temperature control and structure improvement such as set the coil, light source and other optical devices under stable working condition, temperature drift modeling and error compensation measures are proposed to improve FOG's accuracy based on the temperature characteristics of gyro's output [2].

From the study of complex mechanism of FOG, we can see that the temperature variation will cause different refractive index of optical fiber in every sector of the fiber coil and the thermal nonreciprocity effect will be induced; the thermal effect had been described by Shupe and is known as Shupe effect [3]. The thermal nonreciprocity is the important sources of temperature drift and the analysis of FOG's temperature characteristic is helpful to regulate and control the zero drift [4].

As for modeling the nonlinear question of describing the FOG output between temperature gradients, many methods have been proposed such as the time-sequence autoregressive and moving average model [5], the adaptive compensation model [6], and the neural network $[7,8]$. Due to the fact that the neural network has the advantage of approximating the nonlinear function in any expected precision theoretically and has got more and more attention, back-propagation (BP) neural network is an error back-propagation algorithm and can approximate not only all functions but also all step derivatives of them at any given precision. However, because of its easy convergence to a local minimum, slow convergence rate, and the learning progress which limited its engineering application, many researchers have introduced other methods and optimization algorithms to tackle the limitations mainly including network structure modification and learning algorithm innovation, such as genetic algorithm 
(GA) [9], genetic simulated annealing algorithm, and particle swarm optimization (PSO) [10] and the processing results proved that the optimized models are effective and can improve the system's performance. So some other global optimizing algorithms are considered to tune the artificial neural network.

Since the artificial fish swarm algorithm (AFSA) firstly proposed by Li et al. [11] has been applied to many aspects successfully $[5,12,13]$, the AFSA and BP neural network are firstly integrated to describe and model the FOG temperature drift. The algorithm can make good use of the advantages of the two algorithms, so theoretically it will have a better performance. In particular, the AFSA can figure out the global external to overcome the limitation of trapping into local solution of BP neural network. In the tuning procedure, the weights and thresholds of BP neural network can be optimized based on FOG's output, and a greater range of parameters can improve the convergence speed and precision of the algorithm. In the paper, FOG's outputs were obtained under a series of temperature change experiment. In order to improve the modeling performance of the neural network and reduce the random drift in FOG output, the wavelet package algorithm was applied to reprocess the datum [14] and then the AFSA-BP neural network is applied to model the FOG temperature characteristics; at last the compensation result of temperature drift can be used to validate effectiveness of the proposed algorithm.

The paper is organized as follows. Theory analysis of FOG temperature effect and the temperature experiments are described in Section 2. Next, AFSA-BP algorithm is addressed and the reasons why and how BP neural network is optimized by the AFSA are explained. In Section 4, FOG's temperature drift modeling and compensation result with AFSA-BP method and the comparison between BP and GA$\mathrm{BP}$ are presented. Besides, the Allan variance is used to analyse FOG's output. Finally, some conclusions are given in Section 5.

\section{Theory and Experiments}

2.1. FOG Temperature Effect. From the analysis of FOG's output, the FOG drift can be divided into two aspects: noises and bias drifts. Noises determine the minimum detectable phase shift, which consist of temperature noise, light source noise, electronic noise, and others. Drifts determine the bias in the output and show the long-term changes characteristic of gyroscope.

In 1980s, Shupe [3] had proved that a varied temperature which occurs to FOG will yield different refractive index of optical fiber in every sector of the fiber coil; the two slight beams produce a slightly different effective optical path; then a nonreciprocal effect which is known as Shupe effect will be presented, which will bring a negligible error to the output and restricted FOG's application. On Sagnac interferometer, when the two interferential light beams, in two counter directions, track separately the same stretch of the fiber of length $L$, the phase shift error caused by temperature effects
$\Delta \Phi_{e}$ is given by [15]:

$$
\Delta \Phi_{e}=\frac{\beta_{0}}{c_{0}} n \frac{\partial n}{\partial t} \int_{0}^{L / 2}(\Delta T(z)-\Delta T(L-z))(2 z-L) d z
$$

where $\beta_{0}=2 \pi / \lambda$ is the propagation constant of light in vacuum, $n$ is the refractive index of optical fiber, $c_{0}$ is the speed of light in waveguide, and $\Delta T(z)$ is temperature change quantity in the $z$ point of the fiber coil.

2.2. Modeling and Compensation of Temperature Drift. A majority of the modeling and compensation methods to deal with the accuracy improvement of FOG are through using the model to fit the output characteristics of temperature drift data. Most modeling ideas are based on IEEE standard (IEEE Std 952-1997) [16] which including the model equation (2) for a single-axis FOG described the relationship between the input rotation rate and the FOG output:

$$
S_{0}\left(\frac{\Delta N}{\Delta t}\right)=[I+E+D]\left[1+10^{-6} \varepsilon_{k}\right]^{-1},
$$

where $S_{0}$ is nominal scale factor $(" / \mathrm{p}),(\Delta N / \Delta t)$ is output pulse rate $(\mathrm{p} / \mathrm{s}), I$ is inertial input terms $\left({ }^{\circ} / \mathrm{h}\right), E$ is environmentally sensitive terms $\left({ }^{\circ} / \mathrm{h}\right), D$ is drift terms $\left({ }^{\circ} / \mathrm{h}\right)$, and $\varepsilon_{k}$ is scale factor error terms (ppm).

The $E$ represents the drift error caused by environment temperature and can be expressed as

$$
E=D_{T} \cdot \Delta T+D_{\dot{T}} \cdot \frac{d T}{d t}+\bar{D}_{V \overline{\bar{T}}} \cdot \frac{d \nabla \bar{T}}{d t},
$$

where $D_{T}$ is bias drift-rate temperature sensitivity coefficient, $D_{\dot{T}}$ is the coefficient of the temperature-ramp drift-rate sensitivity, and $\bar{D}_{V \dot{\bar{T}}}$ is the coefficient vector of the timevarying temperature-gradient drift-rate sensitivity.

When a certain temperature change happened to FOG, the nonreciprocal phase noises can be calculated, so we should pay more attention to the bias drifts [17]. However, the temperature drift errors compensation is different from the improvement of device structure. Many existing researches have proved the effectiveness of the purely mathematical approach. In this paper, we mainly focus on the influence of temperature and change rate.

2.3. Temperature Experiments. In the experiment, an interferometric FOG is installed on a stationary base which has a temperature box; FOG's static output is acquired under different change rates. The sampling frequency is $100 \mathrm{~Hz}$, and sampling time is 40 minutes. Two sets of temperature change rate are within range of $-5^{\circ} \mathrm{C} / \mathrm{min}$ to $5^{\circ} \mathrm{C} / \mathrm{min}$ and $-8^{\circ} \mathrm{C} / \mathrm{min}$ to $8^{\circ} \mathrm{C} / \mathrm{min}$; the sampling interval of FOG's output and temperature sensors' output are set at 1 second.

Besides, in order to improve the modeling performance of the neural network and reduce the randomness parts in FOG output, we cited wavelet transform method and other preprocessing steps to eliminate constant drift and trend extraction in FOG output [14]; the temperature and denoising results under $\pm 5^{\circ} \mathrm{C} / \mathrm{min}$ are shown in Figure 1 . 

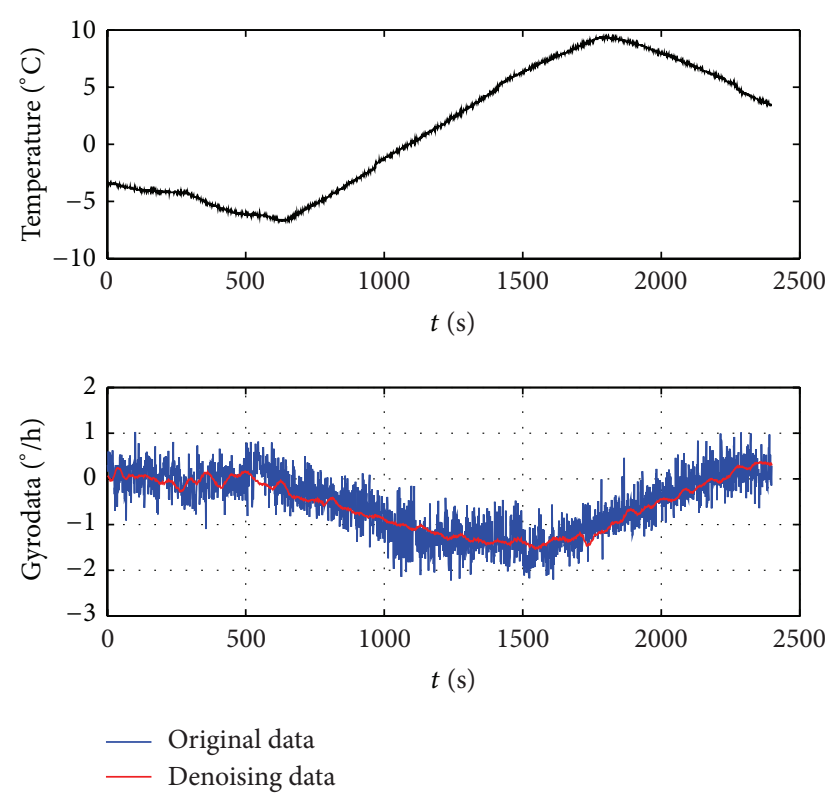

FIGURE 1: FOG original and denoising data under $\pm 5^{\circ} \mathrm{C} / \mathrm{min}$.

\section{AFSA-BP Algorithm}

3.1. Artificial Fish Swarm Algorithm. The AFSA is a novel global optimization algorithm which was inspired by the natural social behavior of fish swarm in searching, swarming, and following. Each individual fish can search its own local optimum and pass on information in the fish swarm, and finally the swarm will achieve a global optimum. Its main feature is parallel processing, independent of initial values, avoidance of converging to a local minimum, and fast training and convergence speed. The related concepts and mathematic description are as follows.

Suppose that the searching space is $D$-dimensional and there are $n$ fishes in the colony. The current state of an artificial fish is $X=\left(X_{1} X_{2} \cdots X_{n}\right)$, where $X_{i}(i=1,2, \ldots, n)$ is variable values of $n$ fishes. The food consistence of $X$ is $Y=$ $f(X)$, where $Y$ is the objective function. The distance between artificial fish $X_{i}$ and $X_{j}$ is $d_{i j}=\left\|X_{i}-X_{j}\right\|$. Defining the sensing distance of artificial fish as Visual and the moving step length of artificial fish as Step, $\delta$ is crowded degree factor [11].

Prey Behavior. The current artificial fish is $X_{i}$, and a randomly select new state is $X_{j}$ in its Visual area. If $Y_{i}<Y_{j}$, move a step in that direction; otherwise, select a $X_{j}$ randomly again and judge whether it satisfied the forward condition. After several random searches, no satisfied conditions then move a step randomly.

Swarm Behavior. Suppose $X_{i}$ is the current state of artificial fish seeking the companion's number $n_{f}$ and their central position $X_{c}$ in its current neighborhood $\left(d_{i j}<\right.$ Visual); if $n_{f}<$ $\delta \cdot N$ and $Y_{i}<Y_{c}$, it means that, at the center of fish colony, there is enough food and the place is not crowded. Then artificial fish move forward the partner center; otherwise do prey behavior.
Follow Behavior. The current artificial fish state $X_{i}$ searches for companion $X_{\max }$ in the neighborhood ( $d_{i j}<$ Visual) with $Y_{\max }$; if $n_{f}<\delta \cdot N$ and $Y_{i}<Y_{\max }$, this represents the $X_{\max }$ has a higher food consistence and is not crowded. The artificial fish will move a step forward companion $X_{\max }$; otherwise, continue to prey behavior.

Random Behavior. After evaluating the current environment of the artificial fish and choosing a state in neighborhood, go forward the direction; it is a default behavior of prey. When repeated numbers are smaller, the artificial fish will forward a random step.

Bulletin Board. Bulletin is used as a board to record the artificial fishes' optimal state and the final optimization state. Each generation artificial fish compares and updates its own state with the bulletin state and decides the next behavior. If the current state is better, the value on the board will be replaced.

The evaluation criterion is based on the problem that we are to address; the usual method is to judge whether the value of MSE repeatedly is less than its allowable error and cannot exceed the extreme of visual and numbers. The MSE of the actual value $y_{i}$ and the predicted value $\hat{y}_{i}$ are expressed as (4), where the sample number is $n$ :

$$
\mathrm{MSE}=\frac{1}{n} \sum_{i=1}^{n}\left(y_{i}-\widehat{y}_{i}\right)^{2} .
$$

3.2. AFSA-BP Algorithm. Based on the advantages of AFSA, we used AFSA as the learning algorithms to determine the parameters of BP neural network. In the training step, construct the artificial fish individuals and take the MSE of the neural network's output as the food consistence of current state.

The structure of three-layered BP neural network is shown in Figure 2. It consists of input, output, and hidden layer; the number of input and output nodes is $n$ and $m$; the hidden nodes are set as two numbers of input nodes. Take the Sigmoidal function as the activation function and calculate the neural network output of every input sample; the MSE of the output is the optimized criterion. When the result meets the minimum of MSE, the weights and thresholds are the best.

In order to construct the artificial fish model, we set the size of artificial fish scale as $N$; take every artificial fish $X_{i}$ as a neural network and two artificial fishes' sum $\left(X_{i}+X_{j}\right)$ or minus $\left(X_{i}-X_{j}\right)$ also as a neural network; the optimized parameters are $\left[w_{i j}\right]$ and $\left[v_{j k}\right] ; w_{i j}$ is weights between $j$ th hidden node and $i$ th input node; $v_{j k}$ is weights between $k$ th output node and $j$ th hidden node; thresholds variables are $\left[w_{j o}\right]$ and $\left[v_{k o}\right]$, where $w_{j o}$ is $j$ th hidden node threshold and $v_{k o}$ is $k$ th output node threshold; all the above parameters are updated after every iteration. After several optimizing steps, the bulletin board will record the final global optimization and the BP neural network with best weights and threshold parameters is determined.

The AFSA-BP algorithm optimizing steps can be described as follows. 


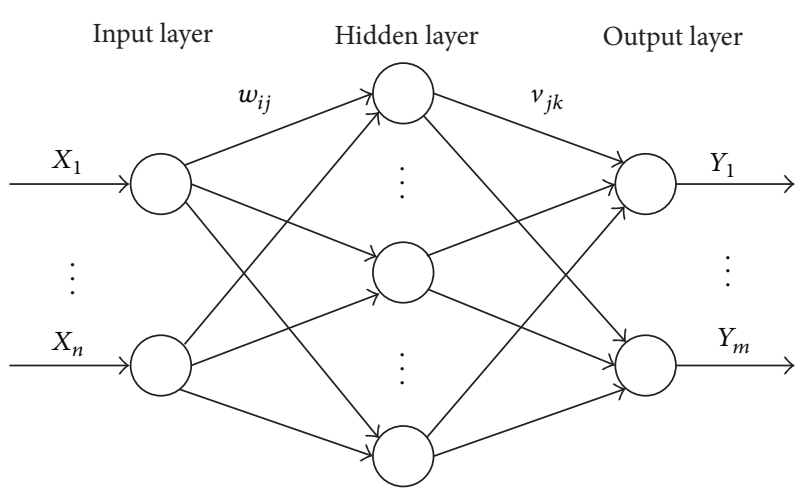

FIGURE 2: Structure of three-layer BP neural network.

(1) Initialize the BP neural network: three layers, $I$ nodes of input layer, $J$ nodes of hidden layer, and $K$ nodes of output layer.

(2) The optimized weights and threshold constitute the matrix $X$ which has the dimension of artificial fish swarm:

$$
\begin{array}{r}
X=X\left(w_{11}, \ldots, w_{I 1}, w_{1 o}, \ldots, w_{1 J}, \ldots, w_{I J}\right. \\
\left.w_{J o}, v_{11}, \ldots, v_{J 1}, v_{1 o}, \ldots, v_{J K}, v_{K o}\right) .
\end{array}
$$

(3) Parameters of artificial fish swarm initialization including pop-size, Visual, Step, $\delta$, and maximum iteration times are Max-Gen, target value $\varepsilon$, and others.

(4) Set the initial number of iteration Gen as 0 , and generate the pop-size artificial fishes randomly, which also constitute the initialized swarm; all the arguments consist of weights and threshold and are between the range of $(-1,1)$.

(5) Calculate the food consistence of every individual artificial fish; compare all the $Y$ value and store the biggest one in the bulletin board.

(6) The four behaviors mentioned above are applied to the fish swarm; each fish simulates the follow and swarm behavior and choose the behavior with bigger $Y$ to continue, the default behavior being prey behavior.

(7) After each iteration, the value of bulletin board is updated when meeting the conditions.

(8) Judge the end condition. When Gen reaches the MaxGen or the MSE meets the target value $\varepsilon$, export the output value $Y$; or Gen pluses one and jump to (6).

The flowchart of AFSA-BP algorithm is shown in Figure 3.

\section{FOG's Temperature Drift Modeling}

4.1. Modeling and Compensation Result. In order to compare the performance of the different modeling methods which

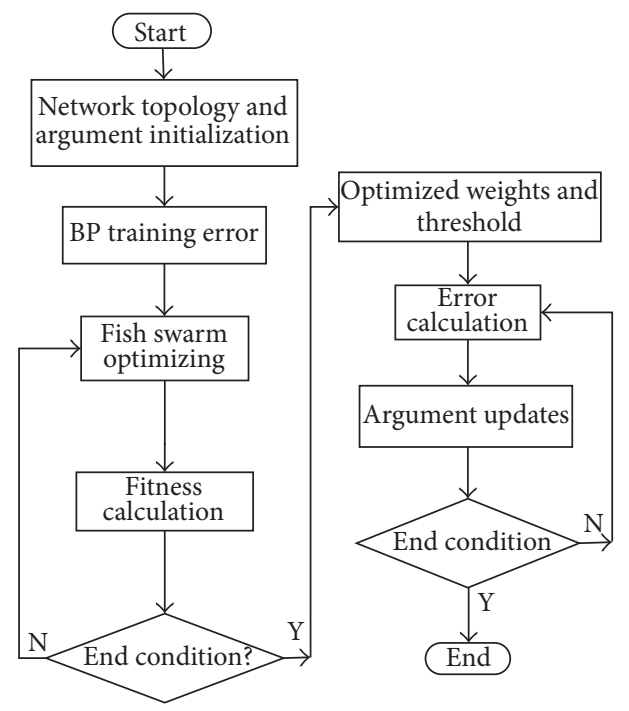

Figure 3: The flowchart of AFSA-BP.

are mentioned and cited in this paper, different from the normal methods of comparing the accuracy when the iterations increased, we take the FOG output data under varied temperature as the input data of models. The different applied methods are BP neural network and GA optimized BP neural network which contain crossover and mutation operation and the AFSA-BP algorithm. By comparing the prediction error curves after training and from the curve figure we can easily identify the algorithm which has better superiority and predict accuracy.

The FOG temperature drift model is based on the AFSA$\mathrm{BP}$ algorithm which is mentioned above; the input of the model is temperature and FOG's temperature drift after denoising processing. Besides, a comparison of the modeling and compensation result with BP neural network and GA optimized BP neural network is expressed. The training data of AFSA-BP neural network is FOG temperature drift data under $\pm 5^{\circ} \mathrm{C} / \mathrm{min}$; the test and predicted data is $\mathrm{FOG}$ temperature drift data under $\pm 8^{\circ} \mathrm{C} / \mathrm{min}$; the prediction and error comparison result of the three algorithms is shown in Figure 4. In the figure, AFSA represents the AFSA-BP neural network.

In order to have a quantitative evaluation of compensation result of FOG's temperature drift, the Allan variance method was applied to analyse the drift compensation result. Allan variance method is a time essential analysis technique and has advantages in evaluating and identifying random noise coefficients [18], such as quantification noise $(Q)$, angle random walk $(N)$, bias instability $(B)$, rate random walk $(K)$, and angular rate ramp $(R)$. It is the standard FOG performance analysis method which is generally acknowledged by IEEE and has been widely used in the gyroscope performance analysis.

The analysis result is shown in Table 1 . The comparison result illustrated the main error sources and the sinusoidal noises in the FOG output signal are reduced by about $50 \%$. 


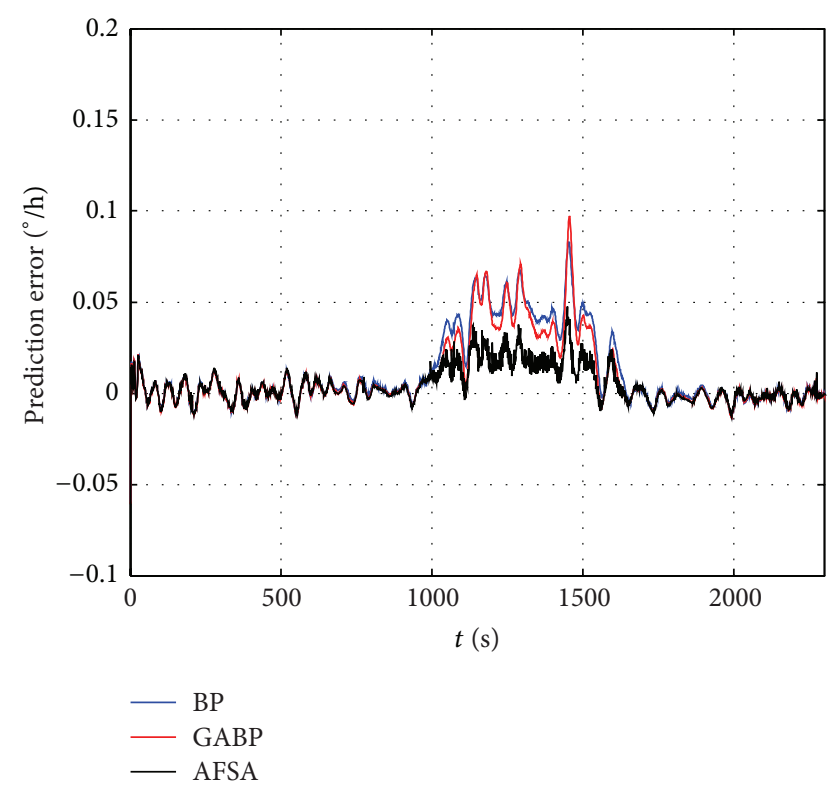

FIGURE 4: Compensation comparison results of the three algorithms.

TABLE 1: Allan variance analysis of FOG's output.

\begin{tabular}{lcccc}
\hline & $\begin{array}{c}\text { Original } \\
\text { data }\end{array}$ & After denoising data & \multicolumn{2}{c}{ Compensation result } \\
& & GABP & AFSA \\
\hline $\mathrm{Q} / \mu \mathrm{rad}$ & 149.37 & 129.58 & 1.48 & 1.17 \\
$N /\left({ }^{\circ} / \mathrm{h}^{1 / 2}\right)$ & 0.94 & 0.79 & 0.009 & 0.007 \\
$B /\left({ }^{\circ} / \mathrm{h}\right)$ & 28.09 & 24.52 & 0.14 & 0.09 \\
$K /\left({ }^{\circ} / \mathrm{h}^{3 / 2}\right)$ & 176.34 & 154.15 & 3.64 & 1.72 \\
$R /\left({ }^{\circ} / \mathrm{h}^{2}\right)$ & 229 & 197.57 & 5.12 & 2.45 \\
\hline
\end{tabular}

Figure 5 is the Allan standard deviation curves of FOG output with the proposed algorithm and other normal methods after compensation. From the figure we can see that the proposed modeling method is effective to improve the FOG precision.

\section{Conclusion}

In this paper, a new hybrid algorithm BP neural network optimized by AFSA is presented and used to describe the temperature drift characteristic of FOG. First, the theory about Shupe effect of FOG was introduced; then the AFSA is used to determine the linking weight and threshold of BP neural network and in order to validate the effectiveness of the method, a set of temperature experiments of FOG under $\pm 5^{\circ} \mathrm{C} / \mathrm{min}$ and $\pm 8^{\circ} \mathrm{C} / \mathrm{min}$ are implemented. Finally, the correlation between FOG's outputs and the parameters of temperature drift model are used to determine the best model. The results of prediction error and curve fitting of different algorithms were presented and can be used to give reference to practical application.

In order to verify the effectiveness of our algorithm, the predicted accuracy of three algorithms, BP neural network

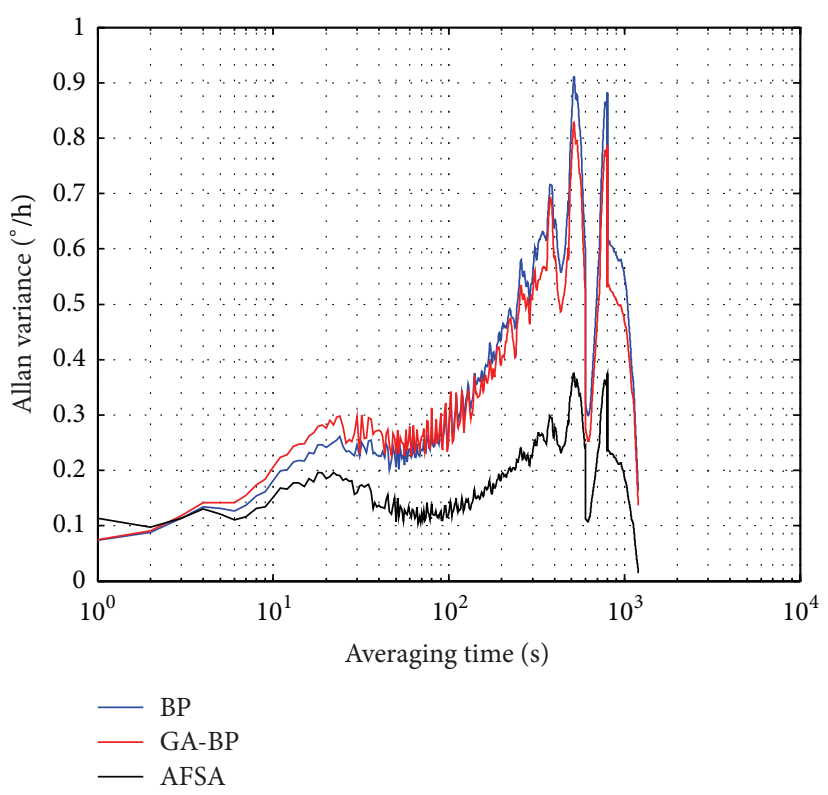

FIGURE 5: Allan variance analysis of the different algorithm FOG output after compensation.

and BP optimized by AFSA and GA, are observed by error prediction curve. Besides, the Allan variance method is applied to get a quantitative evaluation of compensation result of FOG's output before and after a set of processes. The results well validate the main error sources and the sinusoidal noises can be compensated by AFSA-BP neural network and the fiber optic gyroscope precision can be improved.

\section{Conflict of Interests}

The authors declare that there is no conflict of interests regarding the publication of this paper.

\section{Acknowledgments}

This work was supported in part by the National Natural Science Foundation of China (nos. 51375087 and 50975049), Specialized Research Fund for the Doctoral Program of Higher Education (no. 20110092110039), Ocean Special Funds for Scientific Research on Public Causes (no. 20120503509), and the Program Sponsored for Scientific Innovation Research of College Graduate in Jiangsu Province, China (no. CXLX13_083).

\section{References}

[1] H. C. Lefèvre, The Fiber-Optic Gyroscope, National Defense Industry Press, Beijing, China, 2002.

[2] X. Li, C. Zhang, Z. He, and Z. Zhong, "Temperature errors of IFOG and its compensation in engineering application," in Proceedings of the 9th International Conference on Electronic Measurement and Instruments (ICEMI '09), pp. 2230-2234, August 2009. 
[3] D. M. Shupe, "Thermally induced nonreciprocity in the fiberoptic interferometer," Applied Optics, vol. 19, no. 5, pp. 654-655, 1980.

[4] R. Zhu, Y. Zhang, and Q. Bao, "A novel intelligent strategy for improving measurement precision of FOG," IEEE Transactions on Instrumentation and Measurement, vol. 49, no. 6, pp. 1183$1188,2000$.

[5] T. J. Wang, Y. B. Gao, and T. Ma, "Research on FOG random errors modeling based on AFSA," Journal of Chinese Inertial Technology, vol. 20, no. 3, pp. 358-362, 2012.

[6] L. S. Feng, S. Z. Nan, and J. J. Jin, "Research on modeling and compensation technology for temperature errors of FOG," Journal of Astronautics, vol. 27, no. 5, pp. 115-117, 2006.

[7] C. Fan, Z. Jin, W. Tian, and F. Qian, “Temperature drift modelling of fibre optic gyroscopes based on a grey radial basis function neural network," Measurement Science and Technology, vol. 15, no. 1, pp. 119-126, 2004.

[8] Y.Z. Yang and J. T. Fei, "Adaptive sliding mode control of MEMS gyroscope based on neural network approximation," Journal of Applied Mathematics, vol. 2014, Article ID 159047, 8 pages, 2014.

[9] W. L. Yin and Y. H. Tan, "Application of artificial network and genetic algorithm in modeling of fiber optic gyroscope temperature drift," Control and Automation, vol. 22, no. 11, pp. 273-275, 2006.

[10] H. Wang and X. Y. Liu, "Back-propagation neural network training based on particle swarm optimization with best influential partial," Computer Engineering and Application, vol. 43, no. 18, pp. 69-71, 2007.

[11] X. Li, Z. Shao, and J. Qian, "Optimizing method based on autonomous animats: fish-swarm Algorithm," System Engineering Theory and Practice, vol. 22, no. 11, pp. 32-38, 2002.

[12] D. Yazdani, H. Nabizadeh, E. M. Kosari, and A. N. Toosi, "Color quantization using modified artificial fish swarm algorithm," in AI 2011: Advances in Artificial Intelligence, vol. 7106 of Lecture Notes in Computer Science, pp. 382-391, Springer, 2011.

[13] Y. B. Gao, L. W. Guan, and T. J. Wang, "Optimal artificial fish swarm algorithm for the field calibration on marine navigation," Measurement, vol. 50, pp. 297-304, 2014.

[14] G. Xu, W. Tian, Z. Jin, and L. Qian, “Temperature drift modelling and compensation for a dynamically tuned gyroscope by combining WT and SVM method," Measurement Science and Technology, vol. 18, no. 5, pp. 1425-1432, 2007.

[15] B. Han, Y. R. Lin, and Z. I. Deng, "Overview on modeling and compensation of FOG temperature drift," Journal of Chinese Inertial Technology, vol. 17, no. 2, pp. 218-224, 2009.

[16] "IEEE standard specification format guide and test procedure for single-axis interferome tri fiber optic gyros," IEEE Std 9521997, IEEE Aerospace and Electronic System Society, 1997.

[17] X. Chen and C. Shen, "Study on temperature error processing technique for fiber optic gyroscope," Optik, vol. 124, no. 9, pp. 784-792, 2013.

[18] Y. Tang, S. Solve, and T. J. Witt, "Allan variance analysis of Josephson voltage standard comparison for data taken at unequal time intervals," IEEE Transactions on Instrumentation and Measurement, vol. 60, no. 7, pp. 2248-2254, 2011. 

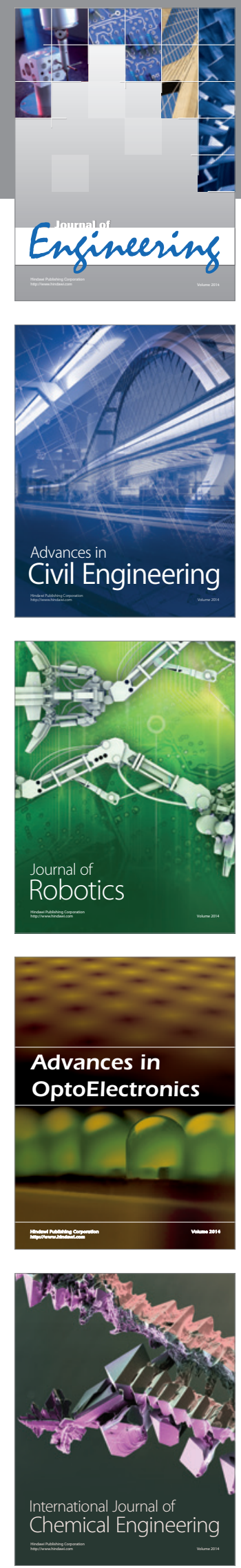

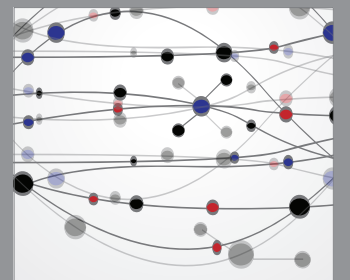

The Scientific World Journal
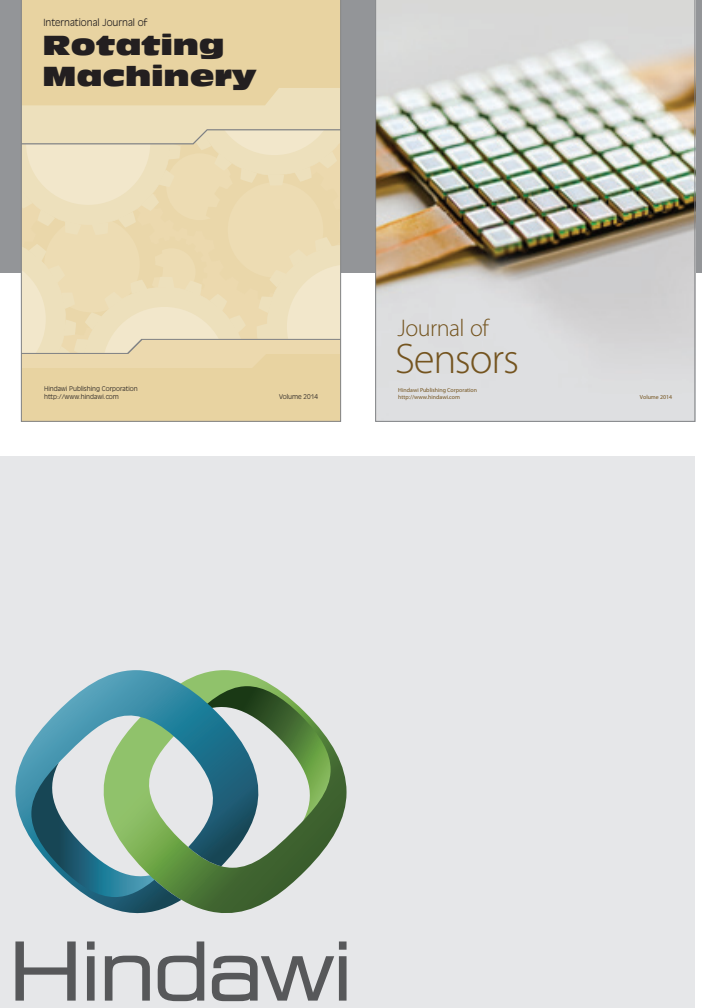

Submit your manuscripts at http://www.hindawi.com
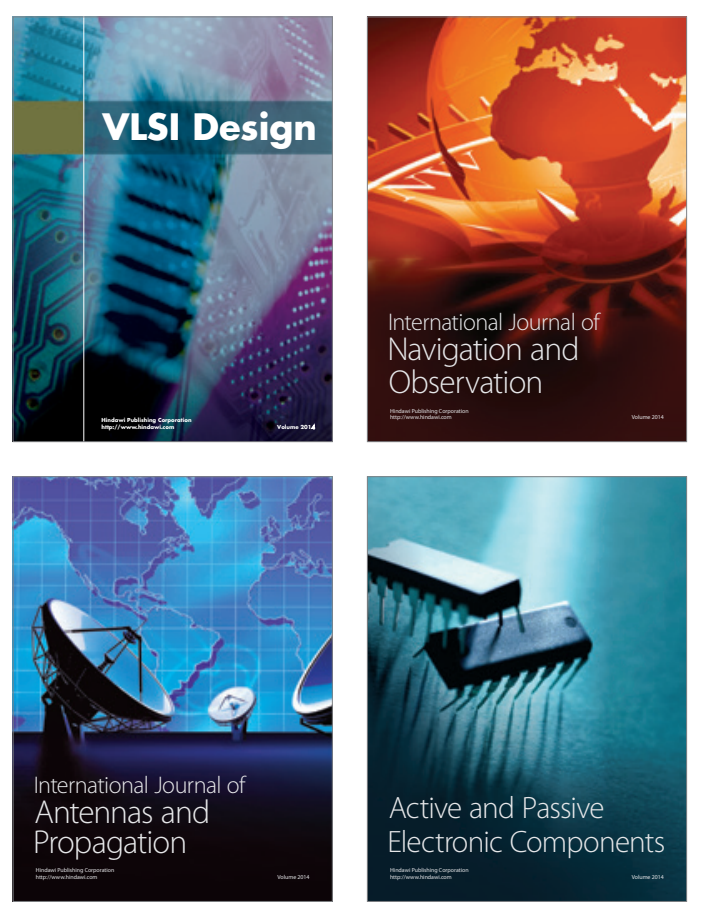
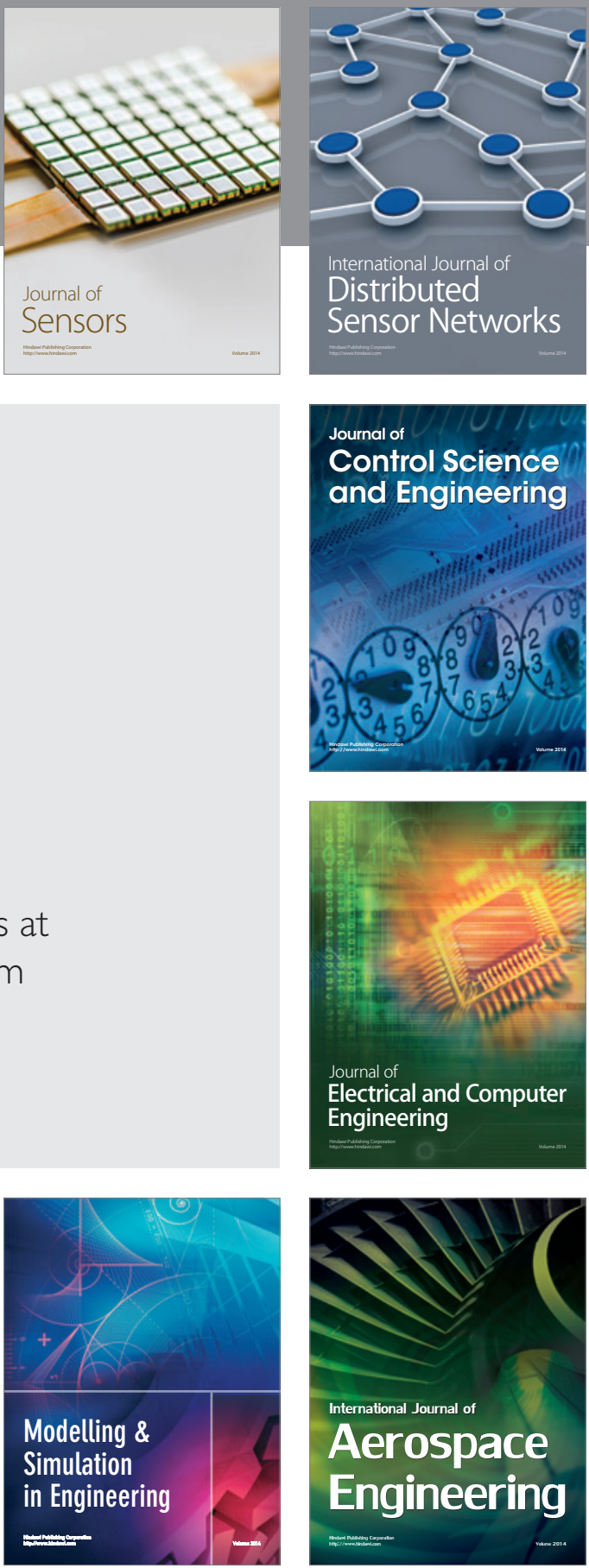

Journal of

Control Science

and Engineering
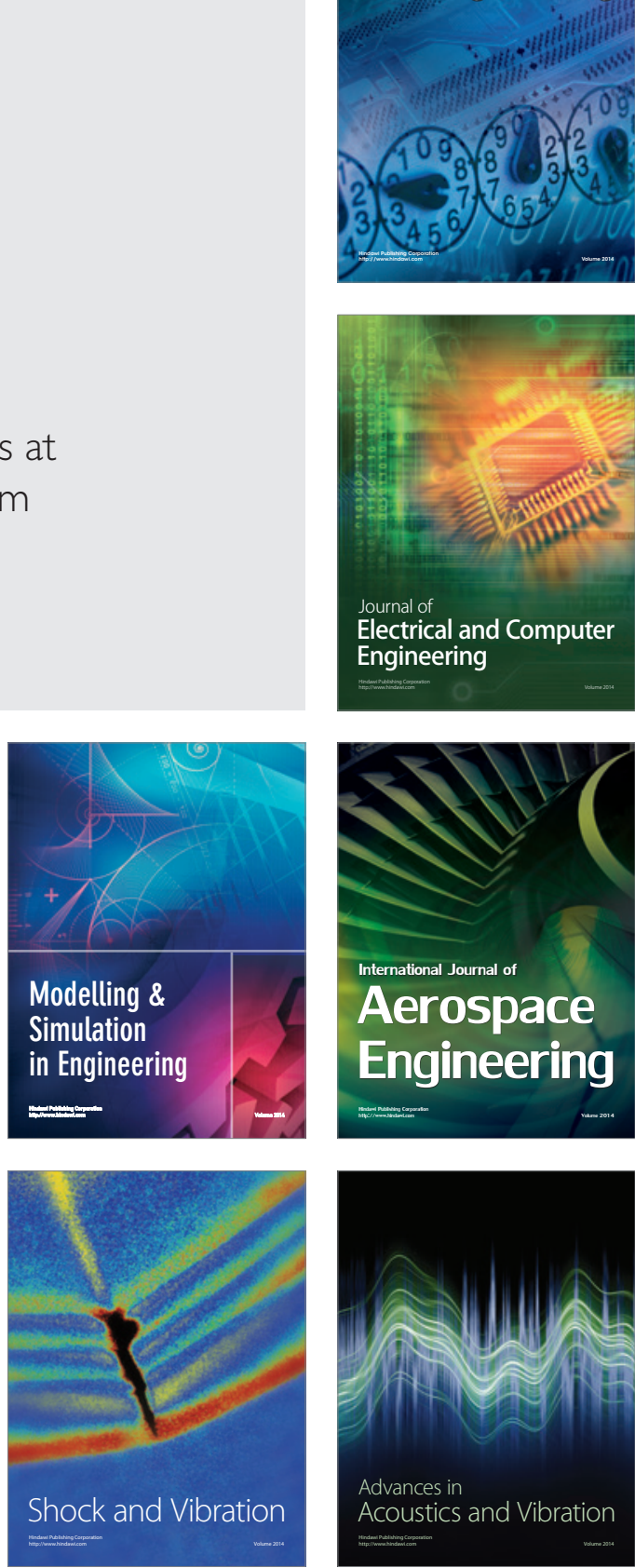